\title{
XXXIV. On the history of the theory of the beats of mistuned consonances
}

\section{R.H.M. Bosanquet}

To cite this article: R.H.M. Bosanquet (1881) XXXIV. On the history of the theory of the beats of mistuned consonances, Philosophical Magazine Series 5, 12:75, 270-282, DOI: 10.1080/14786448108627105

To link to this article: http://dx.doi.org/10.1080/14786448108627105

册 Published online: 28 Apr 2009.

Submit your article to this journal $\sqsubset \pi$

Џ Article views: 3

Q View related articles $\sqsubset$ 
ledged by Mr. Venn ('Symbolic Logic,' p. 271), and practically in the construction of his logical-diagram machine ('Symbolic Logic,'p. 122).

I have constructed a logical-diagram machine on the basis of these diagrams. Blocks, corresponding to the squares or rectangles on the diagrams and resting on two sets of slides, the one set below and at right angles to the other, may be made to drop when the combinations which they indicate are inconsistent with the premises. 32 such slides are required to operate the 256 blocks of an 8-term machine.

But this machine, though simple enough, is practically inferior to the diagrams which $I$ have had printed at a trifling expense for problems of 7, 8, 9, and 10 terms.

XXXIV. On the History of the Theory of the Beats of Mistuned Consonances. By R. H. M. Bosanquet, Fellow of St. John's College, Oxford.

To the Editors of the Philosophical Magazine and Journal. Gentlemen,

TN a paper recently published in the Philosophical Maga1 zine, I have given an account of my recent experiments on an important branch of this subject. I wish to contribute further to the appreciation of the bearing of my results, by means of a critical notice of a few of the most important points connected with the history of the subject; to which I propose to add some remarks on König's recent paper (Annalen der Physik und Chemie, 1880, p. 857).

Passing over the early history of the subject, we come to Smith's 'Harmonics' (1759), a treatise always regarded as important, but difficult. Smith was mainly concerned with the reduction of the phenomena to rule, for the purpose of his studies of musical temperament. His fundamental principles do not really reach beyond the numerical or geometrical appearances or patterns which arise from the superposition of certain sequences of forms at regular intervals. Causal explanation, reference to laws of sensation, analysis of the functions of the ear, were not attempted by Smith. But his work is of great importance. Is is still much appreciated as the first and, in one sense, almost the most powerful exposition of principles still largely held, which are entirely incompatible with the point of view originated by Helmholtz, or its developments.

Freed from the singular phraseology in which Smith's propositions are enveloped, they are not difficult in themselves. I will shortly state in an example the effect of his reasoning, so far as it purports to deal with the causes of beats. 
Smith's 'Harmonics,' prop. x. p. 81, 2nd edit.

We will call a note having 100 vibrations in a certain time a "unison;" and a note having 101 vibrations in the same time will be said to make with the first an "imperfect unison." Then the time in question, in which the notes make respectively 100 and 101 vibrations, is what Smith calls a " simple cycle," or "the period of the imperfect unisons."

Consider notes making 2 and 3 vibrations respectively in the time of 6 of the first note, or " unison." Then the time in which these notes make 2,3 , and 6 vibrations respectively is what Smith calls the "short cycle" of the consonance.

Suppose the consonance to be mistuned, so that 2 vibrations of the one note $=6$ of the unison (100), but three of the other $=6$ of the imperfect unison (101), then the 2 of the one note still very nearly $=3$ of the other, and each of these periods or short cycles is still nearly 6 vibrations both of the unison and the imperfect unison. Then Smith says:-

"Take away the greatest equal numbers of short cycles (of 6) that can be taken from both ends of the simple cycle, or period of the imperfect unisons."

(Take away two sets of 8 short cycles of 6 from 100 and 101 respectively.)

"Then some part of another short cycle or two, as consisting of unequal numbers of the quicker and slower vibrations of the imperfect unisons, will always remain in the middle of the cycle or period."

$(100-2 \times 8 \times 6=4.101-2.8 .6=5$. So that 4 and 5 vibrations respectively are the parts of the short cycles that remain over.)

"And this part, by interrupting the succession of the short cycles" (of 6) "wherein the harmony of the consonance consists, interrupts its harmony, and causes the noise which is called a beat; especially as the interruption is made where the short cycles on each side of it are the most imperfect and inharmonious. Therefore the time between the successive beats, made in the middle of each period or simple cycle of the pulses of the imperfect unisons, or of the least imperfections of the consonance, is equal to the time of the period."

The term period of least imperfections is explained by noticing that there would not be a real coincidence of the two notes at the end of the period; but there would very nearly be one.

This reasoning only points out the mode of calculating the number of times that a certain form of grouping is repeated. So far it is quite right; and as forms of grouping will in this subject always necessarily be a matter of interest and import- 
ance, the modern student cannot afford to dispense with Smith's most powerful exposition. But it does not account for our modern conception of beats, regarded as variations in the intensity of certain notes. We have still to find out in what way such variations of intensity arise out of the system of recurrences Smith describes.

With respect to the supposed difficulty of Smith's propositions, I am confident that any one who has mastered the above example will find the difficulties gone, in all that part of the work which is concerned with the doctrine of beats.

Young's criticism on Smith's work is, that he achieved nothing. De Morgan thinks that Young, and others whom he quotes, did not understand Smith. The passage of Young which De Morgan cites as a proof of this, however, is very good sense, and undoubtedly represents the fact correctly on Young's own theory.

Whether Young understood Smith or not, and whether or no Young's theory will now bear strict criticism, there can be no doubt that Young's work had a most beneficial influence on the progress of the subject. He is remarkably clear about a number of important points; and on some his exposition is not surpassed at the present day.

Young clearly pointed out the mode in which the ordinary beats (which become Smith's short cycles in or near consonances) are numerically related to the Tartini tones (combination-tones). He uses a phraseology which is now seen to be objectionable, when he says that the beats become the Tartini tones. This would involve, in our quantitative language, the statement that the whole of the energy of the beats is transformed into combination-tones, to which statement it is impossible to assent. So that I differ from Young's exposition as I differ from that of König, where he assumes the passage of beats of great frequency into a note, and compares the beats with impulses, as if they contained nothing else but the impulse. But this difference of view will only touch the mode in which the notes arise out of the beats. One might almost say that it is a mere question of language; but it is not so. The element of quantitative transformation is essentially involved.

The facts, however, are of the first importance; and it is not too much to say that Young's statements fully anticipate the principal conclusions of Konnig's great paper, and give the facts completely and compendiously. It is no use attempting a detailed analysis of Young's position, as it is substantially the same as that of König.

Of the other writers who occupy a similar position we need only mention De Morgan, from whose analysis many have 
formed their idea of Smith's work. From our present point of view we admire his painstaking appreciation of Smith, while we think he overestimates the value of Smith's analysis of beats. He does not seem to see that it amounted only to a numerical or geometrical analysis, and left the reader a long way off even the idea of the existence of a deeper-seated explanation. The obstacle opposed to Smith's explanation by the resolution in the ear of the resultant or complex forms into their elements according to Ohm's law, escaped De Morgan entirely.

De Morgan is behind Young in thinking that Tartini (combination) tones only exist in the case of consonances; this vitiates fundamentally all his notions of the phenomena, and causes him to give defective rules for them. Further, he condemns Young for the statement that other resultant tones (as we now call them) arise out of the beats of consonances besides the Tartini tone (difference-tone of Helmholtz, first combination-tone). In fact Young here clearly describes the phenomena subsequently described by König as beat-notes, and was right in the main as to the facts.

I think we may fairly summarize the position taken up by Helmboltz on this question as amounting to the admission of the principle-all beats consist of variations of intensity of musical notes. I am not quite sure whether Helmholtz was the first to enunciate principles which amount to this, as the history of these doctrines is voluminous and rather obscure. And I do not think that even Helmholtz enunciates this as a new dogma in so many words, though it is assumed throughout as the basis of his treatment. Some of the older writers, particularly Smith and Young, so guard their exposition that it is quite capable of adaptation to this principle. On the other hand, certain modern writers, professing or appearing to follow in the footsteps of Smith and Young, so modify their exposition as to make it inconsistent with the above principle, and to require the admission that beats consist of some form of perception other than that of the variation of musical notes.

In treating of the position of Helmholtz, it will be desirable to discuss somewhat minutely a treatise on Beats, consisting of two papers by W. Pole, F.R.S., Mus. Doc., in 'Nature,' Jan. 13 and 20, 1876. I regard these papers as the most important manifesto of the school last alluded to, before the paper of König to be subsequently considered. The clearness and knowledge of the subject make them a good text for discussion; yet I consider the exposition they contain to be erroneous.

Pole describes three kinds of beat-the unison-beat, the 
consonance-beat or Smith's beat, and the beats of Helmholtz, which are taken to include the beats arising from overtones and combination-tones.

The account of the unison-beat is in most respects that common to all authors. But there is a point in which it differs from the carefully-guarded exposition of Smith, which gives rise to a fallacy running throughout the whole paper, though distinct from my principal ground of objection. The whole exposition is founded on effects supposed to be produced by the blows of a hammer. Now this makes a very complicated effect the basis of operations. In the first place, we have in the primary impulse a sound rather than an impulse of the air. This example then involves the question of the production of notes by intermittent sounds, which belongs to a higher and more difficult part of the subject. Secondly, the impulse selected, regarded as an impulse, is of a very complicated character. It can be shown without difficulty that a series of discontinuous impulses is represented by the sum of an infinite series of impulses following the pendulum-law, the amplitudes of all the terms being ultimately equal.

Consider more closely the example given by Pole. Suppose A to make eleven strokes of the hammer to ten of $B$. Then, if at one point the strokes of $A$ and $B$ coincide, after five strokes of B, A will be half a stroke in advance, and the strokes will succeed each other with intervals of half a stroke for a short time; that is to say, the pitch of the resulting sound goes up an octave. Now if two pure tones near each other in pitch produce the unison-beat, the beat consists of alternations of sound and silence very nearly -at all events not of alternations of fundamental and octave. Notes are easily procured of sufficient purity for this demonstration. Consequently Pole's illustration fails to indicate the real nature of the phenomenon.

Smith is careful to avoid this difficulty; and his demonstrations are perfectly applicable to pendulum-vibrations, although he makes no assumption as to the forms of the impulses employed.

The explanation given by Pole of the effect of imperfect coincidences is quite correct; but it is attributed to Young. Now this explanation was fully attained by Smith, and is the origin of the term "period of least imperfections," which is one of the most perplexing elements of his phraseology.

In alluding to the disappearance of unison-beats in intervals greater than a third, Pole notices Young's theory that the beats pass over (? are entirely transformed) into the Tartini tone. But in speaking of the doubt cast by Helmholtz 
on Young's theory, Pole fails to notice the real nature of the discovery of Helmholtz, which is that the two notes are separately received by the ear; so that the disappearance of the beats is to be regarded as arising, in great measure at least, from the removal of the two disturbances from each other's influence, and not entirely or even principally from their rapidity. I have dealt with this point in my recent paper (Phil. Mag. June 1881, p. 422).

Pole's second kind of beat is Smith's beat (variation of maximum displacement in resultant forms). It is assumed that the theory of Smith constitutes a complete explanation of the existence of certain beats in mistuned consonances, as to the nature of which nothing further is said. Smith's investigation has been sufficiently given for its nature to be fully. realized. It will be seen that Smith does not say any thing as to what the ultimate nature of the beat is; he only examines the superposition, and says, "wherever there is a certain irregularity there must be a beat." The classification of beats given by Pole appears therefore to import into Smith's investigation, sub silentio, something not really contained in itnamely, the implication that the beat thus demonstrated is something sui generis, and different in kind from the variation of a musical note. For all beats formed by the variations of musical notes appear to be referred to Pole's third class, viz. the beats of Helmholtz. But before we can admit the existence of beats not consisting of variation of musical notes, we must learn what their sounds really consist of, and have these sounds strictly deduced from the theory; which, to say the least, has not been accomplished so far.

The third kind of beat described by Pole is the overtonebeat of Helmholtz. It is too well understood for any discussion to be needed here.

The combination-tone beats, which were shown in my recent paper to be the actual principal element in the beats of mistuned consonances produced by pure notes, are brought in by Pole at the end, whether regarded as part of the third class or not is not clearly stated.

The next contribution of importance is the famous paper of König on beats and beat-notes, which has been regarded by some authorities as establishing results inconsistent with the views originated by Helmholtz. This paper was to a great extent the origin of my work in connexion with this subject. The question which suggests itself continually in reading König's paper is, "What did all these beats consist of?"-a question which $I$ have endeavoured to answer in my recent paper, and hope to answer more fully on some future occasion. 
276 Mr. R. H. M. Bosanquet on the History of the

König only answers this question once, where he says that, in beats of the mistuned octave, the octave and fundamental appear alternately. I regard this as a proof that König's notes were not free from octave. As to this see further Helmholtz, Tonempfindung, 4th ed. pp. 263, 264; also Preyer, Akustische Untersuchungen (Jena, 1879), p. 15.

On account of the importance of König's paper, I have prepared the following list of the rather numerous errata in the copy in the Philosophical Magazine, as also of those in the copy in Poggendorff. It is not possible to read the copy in the Philosophical Magazine with entire comprehension unless the errata have been first eliminated.

Pogg. Ann. vol. clvii.

$$
\begin{aligned}
& \text { Page } \\
& \text { 191, last line, } \\
& \text { for } m^{\prime}=e \\
& \text { read } m^{\prime}=c
\end{aligned}
$$

192, line 10,

$$
c^{\prime \prime}: g^{\prime \prime}
$$

192, eleven lines from end, for $c^{\prime \prime} e^{\prime \prime}$ read $c^{\prime \prime} c^{\prime \prime}$

193, line 5,

$$
\begin{aligned}
& \text { for } c^{\prime \prime} c^{\prime} \\
& \text { read } c^{\prime \prime} c^{\prime \prime}
\end{aligned}
$$

193, 8 lines from end,

$$
\text { c }
$$

198, 7 lines from end,

$$
\text { C }
$$

194, line 2,

$$
\mathrm{C}: \mathrm{H}
$$

194, line 8,

$$
c^{\prime \prime \prime} h \mathbf{r}
$$

208, line 1,

$$
\text { for } e
$$

read $c$
Phil. Mag. fifth series, vol. i.

Page

427,10 lines from end,

$$
\begin{aligned}
& \text { for } m=e^{\prime} \\
& \text { read } m^{\prime}=c
\end{aligned}
$$

427, 7 lines from end,

$$
c^{n}: d^{n}
$$

427 , last line but one,

$$
\begin{aligned}
& \text { for } e^{\prime \prime}: g^{\prime \prime} \\
& \text { read } c^{\prime \prime}: g^{\prime \prime}
\end{aligned}
$$

428, line 15,

$$
\begin{aligned}
& \text { for } c^{\prime \prime} e^{\prime \prime} \\
& \text { read } c^{\prime \prime} c^{\prime \prime}
\end{aligned}
$$

428, 13 lines from end,

$$
\begin{aligned}
& \text { for } c^{\prime \prime} c^{\prime} \\
& \text { read } c^{\prime \prime} c^{\prime \prime}
\end{aligned}
$$

429, line 13,

$$
\begin{aligned}
& \text { for } c^{\prime} \\
& \text { read } c
\end{aligned}
$$

429, line 14,

$$
\text { for } c
$$

429 , line 22 ,

$$
\begin{aligned}
& \text { for } C: b \\
& \text { read } C: B
\end{aligned}
$$

429 , line 26 ,

$$
\begin{aligned}
& \text { for } c^{\prime \prime \prime}: d_{\mathrm{IV}} \\
& \text { read } c^{\prime \prime \prime}: b_{1 \mathrm{v}}
\end{aligned}
$$

439, line 17 from end, 
Errata (continued).

Pogg. Ann. vol. clvii.

Page

208, line 2,

for $e: c$

read $c: c$

213, line 9 from end,

$c^{\prime}$

213, line 5,

Einklang

Stösse

213 ,

Rauhigkeit u. C ganz achwach Stösse

213,

Octave

Stösse

213,

Duodecime

Stösse

214,

Einklang

Stösse

214,

c deutlich

Stösse

214,

for $c^{\mathrm{IV}}: c^{\mathrm{v}}$

read $c^{\prime \prime \prime}: c^{\nabla}$

215,

Einklang

$$
\text { Stösse }
$$

215 ,

C vernehmbar

Stösse
Phil. Mag. fifth series, vol. i.

Page

439, line 16 from end,

$$
c: c
$$

444, line 8 from end,

$$
\text { for } c
$$

444, line 6,

$$
\begin{aligned}
& \text { for Unison beats } \\
& \text { read Unison. } \\
& \text { Beats. }
\end{aligned}
$$

444, lines 21, 22, 23,

for Roughness and $C$ very weak beats

read Roughness and $\mathrm{C}$ very weak.

Beats.

444, beginning of second period, for octave beats read octave.

Beats.

444 , beginning of third period, for Twelfth beats read Twelfth.

Beats.

445, beginning of first period, for Unison beats read Urison.

Beats.

445 , last line but one of first period,

for $c$ distinct beats

read $c$ distinct.

Beats.

445, heading of third period, for $c^{1 \mathrm{v}}: c^{\mathrm{v}}$ read $c^{\prime \prime \prime}: c^{\nabla}$

446, first line of table, for Unison beats read Unison. Beats.

446, last line but one of table, for $\mathrm{C}$ perceptible beats read $\mathrm{C}$ perceptible. Beats. 


\section{Errata (continued).}

Pogg. Ann. vol. clvii.

Page

216, line 6,

mit den Stosstönen zusammenfallen, und sich also bei diesen nicht nachweisen lassen

216, line 11,

$$
\begin{aligned}
& ,=m \\
& ,=n-m
\end{aligned}
$$

225, - last line of table, for $\mathrm{E}$ read $\mathrm{C}$

232,

beim Zusammenklange zwar periodisch an Intensität zuund abnehmende Schwingungen von nahe zu $\frac{a+b}{2}$ erzeugen.
Phil. Mag. ffth series, vol; i.

Page

511, line 13,

for they coincide with the original note, and therefore cannot be proved by it.

read they coincide with the beat-notes, and therefore cannot be demonstrated beside the latter.

511, line 17, for $m^{\prime}=m$ read The beat-note $=m$

and for $m^{\prime}=n-n$ read The beat-note $=n-m$

517 , last line,

$$
\text { C }
$$

522 , line 21,

for periodically exhibit when sounded together an increase and decrease of vibrations of about $\frac{a+b}{2}$

read when sounded together give rise to vibrations of frequency about $\frac{a+b}{2}$, which periodically increase and decrease in intensity.

The experimental results of König are very completely. stated. So far as they affect the questions in dispute they may be shortly put as follows:-

When any consonance formed by notes having the relation of harmonic and fundamental is mistuned, beats occur.

All such beats* may be regarded as forming beat-notes of corresponding frequency, which become audible when their number and the intensity of the primaries are sufficient.

Two such beat-notes, when nearly forming unison, octave, or twelfth, produce with each other secondary beats. These in turn may be regarded as forming secondary beat-notes.

Difference- and summation-notes are regarded as independent of the beat-notes, and as being much weaker.

Beat-notes are regarded as not susceptible of explanation

* I keep the experimental results clear from König's hypothesis as to their cause. 
by difference- and summation-notes, since the frequencies do not correspond*.

The audibility of beats is regarded as depending solely on their number, and on the intensity of the primaries, and as being independent of the magnitude of the interval. (This is certainly not true; Helmholtz, Tonemp. 4th ed. p. 286.)

In several other respects beats are identified with primary impulses of the same frequency.

According to my results, we should correct these statements by saying that beat-notes are developed from beats by the transformation of a small definite portion of the energy of the beats into the beat-notes, in the transmitting mechanism of the ear.

We now come to a paper of considerable interest, "On Beats of Imperfect Harmonies," by Sir W. Thomson (Proc. Roy. Soc. Edinb. 1877-78, p. 602.) The facts stated in this paper are in accordance with the details of König's experiments. The phase-differences corresponding with the different portions of the beat are exhibited by figures of harmonic curves. The correspondence of the number of beats with that deducible from the forms of the curves is demonstrated experimentally; and the existence of beats is demonstrated in the case of the simpler binary harmonies, when mistuned.

The following is an example of the nature of the description of the sounds heard:- "a revolving character $\dagger$ which I perceive in the beat seems distinct enough to prove that the ear does distinguish between these configurations." . . . It will be seen that the author has here to some extent proposed to himself the question-What are the sounds of which the beats consist? But if my work is right, he has not attained the complete answer to it.

When I first took up the consideration of König's experiments, it seemed to me that the most probable solution was that the notes he used were not pure from overtones. In fact, if we admit the existence of overtones in his notes, phenomena would be produced which would correspond to those he describes. I now know that the beats thus accounted for would not contain some of the elements which do appear. But, since König gives no analysis of the constituent elements of his beats, it was impossible to foresee this. I consequently spent some time in examining the question of the production

* This supposed difficulty arises out of the restriction to first differencetones. In my recent paper the identity of the beat-notes with the various difference-tones is sufficiently established.

$\dagger$ Italics in original, foot of p. 610 . 
of overtones under similar circumstances, and showed that they do exist. Helmholtz (Tonemp. 4th ed. pp. 263, 264) comes to the same conclusion; also Preyer (Akustische Untersuchungen). And it was only by the application of the new methods recently placed at my disposal that I was able to recognize the insufficiency of this explanation, and to place the matter on the footing developed in my recent paper.

It only remains to notice König's paper in Wiedemann's Annalen, 1880, p. 857; and I cannot refrain from expressing my admiration for the clearness and thoroughness with which every point is examined experimentally. I cannot pretend to explain all the phenomena recorded; but I will endeavour to make clear the general point of view from which I regard them.

The proposition König sets himself to maintain is-that a (pure) tone can excite also all the tones of its harmonic series. This proposition was maintained by Wheatstone ; and there is a not inconsiderable number of well-informed persons who are disposed to admit it. Its complete discussion is a matter of very great importance. As a matter of mathematics it is quite inadmissible, unless we admit either the impurity of the "tone" employed or the existence of transformation.

It is generally understood and admitted that a very small excitation, operating on a vibrating body of the same period, is capable of exciting large vibrations in the vibrating body. Suppose, for instance, that in König's pendulum with the spring arrangement at the top (p. 867), the pendulum and weighted spring were arranged to swing in exactly equal periods ; then, how large would the movements of the pendulum have to be to set the spring in vibration with large amplitudes? There can be no doubt that an extremely minute movement of the pendulum, so small as to be scarcely perceptible, would be sufficient for the purpose.

Now, suppose the pendulum arranged as König had it, so that the pendulum swung as a fundamental, and the spring in the period of a harmonic. Then, if the movement of the pendulum contained ever so little of the harmonic in question, the spring would certainly be set in vibration with large amplitudes, just as in the former case; only that, the minute amount of the higher harmonic motion of the pendulum being masked by the fundamental motion, it would appear as if the fundamental itself was exciting the spring in the period of the harmonic. We have then only to inquire, Is it possible that the fundamental vibration of the pendulum can have been accompanied by a small amount of harmonic? And we answer, it is 
not only possible, but certain that it must be so. When we speak of pendulum-vibrations we are apt to forget our history, and to think of the circular pendulum as if it really executed perfect harmonic motions. But this is only true of the ideal cycloidal pendulum, which never has been realized, and is never likely to be. And the difference between the two is not negligible: we need only think of the correction for arc which the periodic time requires in pendulum-observations of accuracy, to see that higher harmonic terms must enter into the motion; and in all cases in which the fundamental vibration is not very small indeed, these higher terms will certainly not be negligible.

With any other experimenter than König I should be disposed to point to a number of influences which lead to transformation. One may possibly have escaped him : it is necessary for his purposes to be sure that the bending of the spring calls into action a force strictly proportional to the displacement. For large displacements it is improbable that this condition is satisfied. Further, any unsteadiness in the stand of the instrument leads to transformations of very considerable extent; but this is not likely to have misled König.

The remaining investigations will now be more easily dealt with.

On pages $860 \& 861$ the argument rests on the idea that the harmonic in the source of excitation must be developed to the same actual magnitude as the excited harmonic vibration. But this is not at all the case. According to both theory and practice, the excited harmonic vibration can be developed by an extremely small corresponding vibration in the exciter; and it seems to me quite probable that a large fundamental may contain sufficient harmonic for the purpose of excitation without showing any trace of it in its curves, as is apparently the case on page 863 .

I do not say that this is the sole explanation of the results; but it is sufficient to prevent me from accepting König's argument as a proof.

There is much difficulty in the question of transmission through the air. When we think of the complicated series of currents and vortices that must surround the prongs of a tuning-fork, it seems very difficult to be sure that there is no transformation there. But certainly König's experiment with the phonantograph (p. 864) seems to refer us back to the explanation above stated.

With respect to the transmission of the vibrations through threads (p. 865), I examined this point some years ago by means of a little apparatus shown to the British Association

Plil. Mag. S. 5. Vol. 12. No. 75. Oct. 1881 . Y 
in 1876. If the threads are not so arranged that the pull on the excited vibrator is strictly proportional to the displacement of the exciter, it is quite certain, both from theory and experiment, that transformation must take place. My apparatus consisted of a number of pendulums arranged as harmonics to a large and heavy metronome. The connexions were made with elastic threads. When the connexions were so made as to satisfy the above requirement as nearly as possible, I could reduce the excitation of the harmonic pendulums to a very small amount; I never succeeded in entirely stopping it. But it was obviously impossible to fulfil the above condition with any approach to real perfection. When, however, the approximate fulfilment of the condition was purposely avoided, as by letting the thread just go slack at one point of the vibration, transformation set in at once, as it should do, and the small pendulums were set in violent vibration. At that time I pointed out the defect of Mayer's arrangement of transmission by threads (note, p. 865), as it did not appear from the account that any means were taken to render the pull strictly proportional to the displacement of the exciter, and consequently transformation was to be expected.

On the whole, no doubt, the truth of the matter is best stated in a form that combines much of what both parties to this discussion have maintained. It is quite true that, in a hypothetical system in which the forces called into action are strictly proportional to the displacements, the fundamental harmonic vibration cannot permanently excite its multiples. This is unassailable as matter of mathematics; and as to experiment, we can only say that, the nearer we approach to the construction of such a system, the less are the multiples excited by the fundamental. But I think that the actual construction of such a system is impossible. And so far as our actual systems depart from the above condition, more or less, transformation does and must take place. Wheatstone's law is therefore generally true of actual systems ; and it is only incomplete because it omits the question of quantity. How much of the multiple vibration is excited in systems whose forces depart to known extents from the simple law of proportionality? So far as we are able to answer by our general knowledge of the facts, they are entirely in accordance with the theory. "The more minute comparison of the different classes of systems should be an experimental study of great interest but no theoretical difficulty, which would materially assist to throw light on the general comprehension of the subject. 